\title{
Variation in Topical Antibiotics Recommendations in Wound Care Instructions by Non-dermatologists
}

\author{
Ramie Fathy, $A B^{7}$, Brian Chu, $B S^{7}$, Partik Singh, $M D, M B A^{2}$, William D. James, $M D^{3}$, and \\ John S. Barbieri, $M D, M B A^{3}(D)$ \\ 'University of Pennsylvania Perelman School of Medicine, Philadelphia, PA, USA; ${ }^{2}$ Northwestern University Feinberg School of Medicine, Chicago, IL, \\ USA; ${ }^{3}$ Department of Dermatology, University of Pennsylvania Perelman School of Medicine, Philadelphia, PA, USA.
}

$\mathrm{J}$ Gen Intern Med 36(1):238-9

DOI: $10.1007 / \mathrm{s} 11606-020-05689-2$

(c) Society of General Internal Medicine 2020

T o the Editors:

Skin biopsies are an important diagnostic tool for dermatologists and other clinicians. Although several studies have demonstrated that topical antibiotics are not useful following uncomplicated skin biopsies, ${ }^{1}$ a recent study found that nearly half of wound care instructions from dermatologists continue to recommend their use. ${ }^{2}$ Given that skin biopsies are also performed by clinicians in other specialties such as internal medicine and family medicine, particularly in areas where dermatologists are scarce, the purpose of this study was to evaluate wound care instructions by non-dermatologists.

We identified the top non-dermatologist utilizers of skin biopsies in the 2017 Medicare Public Use File. Only allopathic and osteopathic physicians were included. Physicians were excluded if they were boarded in dermatology or lacked a practice-associated webpage. Webpages were identified by conducting a web search using each physician's name and address. Each webpage was subsequently reviewed to identify written instructions for post-biopsy wound care (e.g., patient handouts or FAQs). This process was continued among the most frequent utilizers of skin biopsies with the goal of reaching a sample size of 25 sets of wound care instructions.

Of the top 259 non-dermatologist utilizers of skin biopsies, $202(78 \%)$ were identified to have a webpage associated with their practice, 25 (10\%) of which included post-biopsy wound care instructions. Of the 25 sets of wound care instructions identified, 10 explicitly recommended topical antibiotics (40\%). The most commonly mentioned topical antibiotics were bacitracin (5), polysporin (4), and mupirocin (1). Hydrogen peroxide was mentioned in 3 instructions (12\%), while

Ramie Fathy and Brian Chu contributed equally to this work.

Received January 21, 2020

Accepted January 28, 2020

Published online February 3, 2020 non-antibiotic ointments, including petrolatum-based products, were mentioned in 7 instructions (28\%). Eight instructions $(32 \%)$ did not include any recommendations for specific topical wound care products (Table 1). Notably, no resource explicitly advised against the use of topical antibiotics.

Based on data from several randomized controlled trials, topical antibiotics do not reduce the risk of surgical site infection and are associated with increased risk of antibioticassociated complications such as contact dermatitis, antibiotic resistance, and impaired wound healing. 1, 3, 4 The American Academy of Family Physicians guidelines recommend against the use of topical antibiotics. ${ }^{5}$ Similar to what has previously been observed among dermatologists, our results highlight that wound care instructions from other clinicians also frequently recommend the use of topical antibiotics. ${ }^{2}$ Given the high frequency of topical antibiotic recommendation among wound care instructions, there is likely an opportunity to improve the educational materials provided to patients.

The findings of this study should be interpreted in the context of the study design. This study is limited to information available online, which does not include physical instructions provided to patients in the clinic or information discussed elsewhere during verbal counseling. Future studies are needed to evaluate whether our findings generalize to other communication modalities and settings. Nevertheless, these findings illustrate a substantial variability in post-biopsy antibiotic recommendations and a persistence of topical antibiotic promotion despite strong evidence opposing it. ${ }^{5}$ There is a need for increased awareness of the low value of topical antibiotic use following uncomplicated cutaneous procedures.

Table 1 Recommendations of Topical Wound Care Agents Following Skin Biopsies, as Described in Wound Care Instructions

\begin{tabular}{ll}
\hline \hline & $N(\%)$ \\
\hline All instructions & 25 \\
Recommended topical antibiotics & $10(40 \%)$ \\
Bacitracin & $5(20 \%)$ \\
Polysporin & $4(16 \%)$ \\
Mupirocin & $1(4 \%)$ \\
Recommended hydrogen peroxide & $3(12 \%)$ \\
Recommended non-antibiotic ointments & $7(28 \%)$ \\
Did not recommend specific topical products & $8(32 \%)$ \\
\hline
\end{tabular}


Corresponding Author: John S. Barbieri, MD, MBA; Department of Dermatology University of Pennsylvania Perelman School of Medicine, Philadelphia, PA, USA (e-mail: john.barbieri@pennmedicine.upenn. edu).

Funding Information Dr. Barbieri is financially supported by the National Institute of Arthritis and Musculoskeletal and Skin Diseases of the National Institutes of Health under award number T32-AR-007465 and receives partial salary support through a Pfizer Fellowship in Dermatology Patient Oriented Research grant to the Trustees of the University of Pennsylvania.

\section{Compliance with Ethical Standards:}

Conflict of Interest: The authors declare that they do not have a conflict of interest.

Disclaimer: The funding sources had no role in the design and conduct of the study; collection, management, analysis, and interpretation of the data; preparation, review, or approval of the manuscript; and decision to submit the manuscript for publication.

\section{REFERENCES}

1. Saco M, Howe N, Nathoo R, Cherpelis B. Topical antibiotic prophylaxis for prevention of surgical wound infections from dermatologic procedures: a systematic review and meta-analysis. J Dermatol Treat. 2014;26(2):151158. https://doi.org/10.3109/09546634.2014.906547.

2. Nguyen JK, Huang A, Siegel DM, Jagdeo J. Variability in Wound Care Recommendations Following Dermatologic Procedures. Dermatol Surg. 2019.

3. Totoraitis BS, Cohen JL, Friedman A. Topical Approaches to Improve Surgical Outcomes and Wound Healing: A Review of Efficacy and Safety. J Drugs Dermatol. 2017; 16(3): 209-212.

4. Smack DP, Harrington AC, Dunn C, et al. Infection and allergy incidence in ambulatory surgery patients using white petrolatum vs bacitracin ointment. A randomized controlled trial. JAMA. 1996;276(12):972-977.

5. Pickett H. Shave and punch biopsy for skin lesions. Am Fam Physician. 2011;84(9):995-1002.

Publisher's Note Springer Nature remains neutral with regard to jurisdictional claims in published maps and institutional affiliations. 